International Journal of Pure and Applied Mathematics

Volume 93 No. 5 2014, 729-739

ISSN: 1311-8080 (printed version); ISSN: 1314-3395 (on-line version)

url: http://www.ijpam.eu

doi: http://dx.doi.org/10.12732/ijpam.v93i5.11

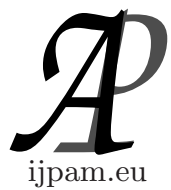

\title{
THIRD NOTE ON THE SHAPE OF $S$-CONVEXITY
}

\author{
I.M.R. Pinheiro \\ P.O. Box 12396, A'Beckett St \\ Melbourne, Victoria, AUSTRALIA, 8006
}

\begin{abstract}
As promised in Second Note on the Shape of $S$-convexity, we now discuss the exponent of the piece of definition for $S$-convexity that deals with negative images of real functions. We also present a severely improved definition for the phenomenon.
\end{abstract}

AMS Subject Classification: 26A51

Key Words: analysis, convexity, definition, $S$-convexity, geometry, shape

\section{Introduction}

In First Note on the Shape of $S$-convexity, we confirmed the value of our rewording of the piece of definition for the phenomenon $S$-convexity that deals with non-negative real functions and we proposed a geometric definition for the phenomenon.

In Second Note on the Shape of $S$-convexity, we confirmed that our added piece of definition for the phenomenon $S$-convexity, that for negative real functions, is a proper extension of Convexity and we proposed a geometric definition for that case.

Still in our second note, we observed that our limiting curve for $S$-convexity, when the real function is negative, is a bit bigger than the limiting curve for $S$-convexity when the real function is not negative in terms of length, what means that our lift is not the same for both cases.

Received: April 14, 2014

(c) 2014 Academic Publications, Ltd. url: www.acadpubl.eu 
Our perimeters are still close enough: The discrepancy appears by the first decimal digit only and is less than 0.5 in dimension (considering our approximation for $p i$, our manual calculation, and the approximation for the perimeter via elliptical curve or circumference).

In Third Note on the Shape of $S$-convexity, we study the perimeter of our limiting curve for the phenomenon $S$-convexity aiming at equal perimeters for situations that could be seen as similar, geometrically speaking.

As a consequence, we here propose new refinements to the definition of the phenomenon.

Pieces of the analytical definition of the phenomenon $S$-convexity that we here deal with ([1] and [2])

Definition 2. A function $f: X \rightarrow \Re$, where $|f(x)|=f(x)$, is told to belong to $K_{s}^{2}$ if the inequality

$$
f(\lambda x+(1-\lambda)(x+\delta)) \leq \lambda^{s} f(x)+(1-\lambda)^{s} f(x+\delta)
$$

holds $\forall \lambda / \lambda \in[0,1] ; \forall x / x \in X ; s=s_{2} / 0<s_{2} \leq 1 ; X / X \subseteq \Re_{+} \wedge X=[a, b]$; $\forall \delta / 0<\delta \leq(b-x)$.

Definition 3. A function $f: X \rightarrow \Re$, where $|f(x)|=-f(x)$, is told to belong to $K_{s}^{2}$ if the inequality

$$
f(\lambda x+(1-\lambda)(x+\delta)) \leq(1-\lambda)^{\frac{1}{s}} f(x)+\lambda^{\frac{1}{s}} f(x+\delta)
$$

holds $\forall \lambda / \lambda \in[0,1] ; \forall x / x \in X ; s=s_{2} / 0<s_{2} \leq 1 ; X / X \subseteq \Re_{+} \wedge X=[a, b]$; $\forall \delta / 0<\delta \leq(b-x)$.

Remark 1. If the inequality is obeyed in the reverse ${ }^{1}$ situation by $f$, then $f$ is told to be $s_{2}$-concave.

Pieces of the geometrical definition that we here deal with ([3] and [4])

Definition 4. A real function $f: X \rightarrow Y$, for which $|f(x)|=f(x)$, is called $S$-convex if and only if, for all choices $\left(x_{1} ; y_{1}\right)$ and $\left(x_{2} ; y_{2}\right)$, where $\left\{x_{1}, x_{2}\right\} \subset X,\left\{y_{1}, y_{2}\right\} \subset Y, Y=I_{f}$, and $x_{1} \neq x_{2}$, it happens that the line drawn between $\left(x_{1} ; y_{1}\right)$ and $\left(x_{2} ; y_{2}\right)$ by means of the expression $(1-\lambda)^{s} y_{1}+\lambda^{s} y_{2}$, where $\lambda \in[0,1]$, does not contain any point with height, measured against the vertical Cartesian axis, that be inferior to the height of its horizontal equivalent in the curve representing the ordered pairs of $f$ in the interval considered for the line in terms of distance from the origin of the Cartesian axis.

\footnotetext{
${ }^{1}$ Reverse here means $>$, not $\geq$.
} 
Definition 5. A real function $f: X \rightarrow Y$, for which $|f(x)|=-f(x)$, is called $S$-convex if and only if, for all choices $\left(x_{1} ; y_{1}\right)$ and $\left(x_{2} ; y_{2}\right)$, where $\left\{x_{1}, x_{2}\right\} \subset X,\left\{y_{1}, y_{2}\right\} \subset Y, Y=\operatorname{Im}_{f}$, and $x_{1} \neq x_{2}$, it happens that the line drawn between $\left(x_{1} ; y_{1}\right)$ and $\left(x_{2} ; y_{2}\right)$ by means of the expression $(1-\lambda)^{\frac{1}{s}} y_{1}+\lambda^{\frac{1}{s}} y_{2}$, where $\lambda \in[0,1]$, does not contain any point with height, measured against the vertical Cartesian axis, that be inferior to the height of its horizontal equivalent in the curve representing the ordered pairs of $f$ in the interval considered for the line in terms of distance from the origin of the Cartesian axis.

\section{Chasing Equal Lengths for Both Cases}

In [3], we have reached the following expression for the Arc Length of our limiting curve (non-negative real functions):

$$
p \equiv \int_{0}^{1} \sqrt{1+\left[-s(1-\lambda)^{s-1} y_{1}+s \lambda^{s-1} y_{2}\right]^{2}} d \lambda .
$$

In Second Note on the Shape of $S$-convexity, the expression for the Arc Length was (negative real functions):

$$
p \equiv \int_{0}^{1} \sqrt{1+\left[-\frac{1}{s}(1-\lambda)^{\frac{1-s}{s}} y_{1}+\frac{1}{s} \lambda^{\frac{1-s}{s}} y_{2}\right]^{2}} d \lambda .
$$

Our tables, containing samples of values for $s$ and their respective Arc Lengths, had been put together through approximating values to no decimals in the third listed value for $s, 0.25$, and through approximating the value of pi to 3.141516 plus the result of the calculation to two decimal digits in the second listed value for $s, 0.5$.

The negative case is giving more rope than the positive case, since we have 1.2 (non-negative) for one case and 1.0 (negative) for the other.

That should mean that we get more functions in each s-group if we choose to equate the limiting line for the non-negative functions to the limiting line for the negative ones.

To chase equal lengths for both cases, we first observe that if the percentage we select inside of the brackets when the definition of the $S$-convexity limiting line is used is the same, what commands the result is the value of the function, first of all, and then the value of the exponent we raise the percentage to.

This way, to compare both cases and chase the same result, we should start by finding functions that hold the same value in modulus in the interval we choose to consider. 
Whilst, in the non-negative case, we increase the value of the percentage when raising it to a fractionary exponent, given that it is always at most $100 \%$ and at least $0 \%$; in the negative case, we decrease it if doing the same because increasing the portion we take from a negative value is making the result smaller, not bigger, as it happens with the positive value.

That tells the reader why we guessed $\frac{1}{s}$ for the second part of our definition.

To chase equal lengths, we use the convexity limiting line as a reference line for both cases and equate the moduli of the differences between the limiting lines for the extension of convexity and the limiting lines for convexity.

We choose the constant functions to work with because they are the nicest functions in the group of functions available to us inside of the phenomenon.

With this, suppose that $g(x)=A, A>0$ (notice that $A=0$ implies that the limiting line for convexity is equal to the limiting line for $S$-convexity), and $h(x)=-A$.

If we look for an exponent that produce the same effect we get with $\frac{1}{s}$ when we have a negative function, we may call this exponent $\delta_{1}$ and think in the following way:

Suppose that $f\left(\lambda_{c p}\right)=(1-\lambda) A+\lambda A, f\left(\lambda_{s c p}\right)=(1-\lambda)^{\delta_{1}} A+\lambda^{\delta_{1}} A, f\left(\lambda_{c n}\right)=$ $(\lambda-1) A-\lambda A$, and $f\left(\lambda_{s c n}\right)=-(1-\lambda)^{\frac{1}{s}} A-\lambda^{\frac{1}{s}} A$.

We then have $\left|f\left(\lambda_{s c p}\right)-f\left(\lambda_{c p}\right)\right|=\left|f\left(\lambda_{s c n}\right)-f\left(\lambda_{c n}\right)\right|$ and, with this, $\mid(1-$ $\lambda)^{\delta_{1}} A+\lambda^{\delta_{1}} A-[(1-\lambda) A+\lambda A]|=|-(1-\lambda)^{\frac{1}{s}} A-\lambda^{\frac{1}{s}} A-[(\lambda-1) A-\lambda A] \mid$.

What follows is:

$$
\left|(1-\lambda)^{\delta_{1}}+\lambda^{\delta_{1}}-1\right|=\left|-(1-\lambda)^{\frac{1}{s}}-\lambda^{\frac{1}{s}}+1\right| .
$$

Making $\lambda=\frac{1}{2}$ for practical purposes, we have:

$$
\left|2^{-\delta_{1}}+2^{-\delta_{1}}-1\right|=\left|-2^{-\frac{1}{s}}-2^{-\frac{1}{s}}+1\right|
$$

What follows is:

$$
\left|2 * 2^{-\delta_{1}}-1\right|=\left|-2 * 2^{-\frac{1}{s}}+1\right| \text {. }
$$

Then:

(A) $2 * 2^{-\delta_{1}}-1=-2 * 2^{-\frac{1}{s}}+1$ or

(B) $2 * 2^{-\delta_{1}}-1=2 * 2^{-\frac{1}{s}}-1$.

From A, we get:

$$
2^{1-\delta_{1}}-1=-2^{1-\frac{1}{s}}+1
$$




$$
\begin{aligned}
& \therefore \quad 2^{1-\delta_{1}}=2-2^{1-\frac{1}{s}}, \\
& \therefore \quad 2^{\delta_{1}}=\frac{2}{2-2^{1-\frac{1}{s}}}, \\
& \therefore \quad 2^{\delta_{1}}=\frac{1}{1-2^{-\frac{1}{s}}} .
\end{aligned}
$$

A then leads to our $s$ being replaced with $\log _{2}\left(\frac{1}{1-2^{-\frac{1}{s}}}\right)$ and B leads to our replacement being $\frac{1}{s}$.

Because both differences should be non-negative (we are always doing the limiting line of the extension minus the limiting line of convexity), only A is a valid result.

It is then the case that $\delta_{1}=\log _{2}\left(\frac{1}{1-2^{-\frac{1}{s}}}\right)$.

With this, our function is smooth of class $C^{\infty}$ ([5], p. 511).

To calculate our perimeters, we now have to use the formula we had for the negative case and the new formula, which will be a result of our calculations.

Assume that $A=1$.

$$
\begin{aligned}
& f\left(\lambda_{\text {scp }}\right)=(1-\lambda)^{\log _{2}\left(\frac{1}{1-2^{-\frac{1}{s}}}\right)}+\lambda^{\log _{2}\left(\frac{1}{1-2^{-\frac{1}{s}}}\right)}, \\
& f^{\prime}\left(\lambda_{s c p}\right)=-\log _{2}\left(\frac{1}{1-2^{-\frac{1}{s}}}\right)(1-\lambda)^{\log _{2}\left(\frac{1}{1-2^{-\frac{1}{s}}}\right)-1} \\
& +\log _{2}\left(\frac{1}{1-2^{-\frac{1}{s}}}\right) \lambda^{\log _{2}\left(\frac{1}{1-2^{-\frac{1}{s}}}\right)-1} \\
& \left(f^{\prime}\left(\lambda_{s c p}\right)\right)^{2}=\left(-\log _{2}\left(\frac{1}{1-2^{-\frac{1}{s}}}\right)(1-\lambda)^{\log _{2}\left(\frac{1}{1-2^{-\frac{1}{s}}}\right)-1}\right. \\
& \left.+\log _{2}\left(\frac{1}{1-2^{-\frac{1}{s}}}\right) \lambda^{\log _{2}\left(\frac{1}{1-2^{-\frac{1}{s}}}\right)-1}\right)^{2}, \\
& p \equiv \int_{0}^{1}\left(1+\left(-\log _{2}\left(\frac{1}{1-2^{-\frac{1}{s}}}\right)(1-\lambda)^{\log _{2}\left(\frac{1}{1-2^{-\frac{1}{s}}}\right)-1}\right.\right.
\end{aligned}
$$




$$
\left.+\log _{2}\left(\frac{1}{1-2^{-\frac{1}{s}}}\right) \lambda^{\log _{2}\left(\frac{1}{1-2^{-\frac{1}{s}}}\right)-1}\right)\left.^{2}\right|^{\frac{1}{2}} d \lambda .
$$

Let's call this new set of functions New Positive.

Now we try to use the exponent we have for the non-negative case as a model and see what we get $([4])$.

If we look for an exponent that produce the same effect we get with $s$ when we have a non-negative function, we may call this exponent $\delta_{2}$ and think in the following way:

Suppose that $f\left(\lambda_{c p}\right)=(1-\lambda) A+\lambda A, f\left(\lambda_{s c p}\right)=(1-\lambda)^{s} A+\lambda^{s} A, f\left(\lambda_{c n}\right)=$ $(\lambda-1) A-\lambda A$, and $f\left(\lambda_{s c n}\right)=-(1-\lambda)^{\delta 2} A-\lambda^{\delta 2} A$.

We then have $\left|f\left(\lambda_{s c p}\right)-f\left(\lambda_{c p}\right)\right|=\left|f\left(\lambda_{s c n}\right)-f\left(\lambda_{c n}\right)\right|$ and, with this, $\mid(1-$ $\lambda)^{s} A+\lambda^{s} A-[(1-\lambda) A+\lambda A]|=|-(1-\lambda)^{\delta_{2}} A-\lambda^{\delta_{2}} A-[(\lambda-1) A-\lambda A] \mid$.

What follows is:

$$
\left|(1-\lambda)^{s}+\lambda^{s}-1\right|=\left|-(1-\lambda)^{\delta_{2}}-\lambda^{\delta_{2}}+1\right| .
$$

Making $\lambda=\frac{1}{2}$ for practical purposes, we have:

$$
\left|2^{-s}+2^{-s}-1\right|=\left|-2^{-\delta_{2}}-2^{-\delta_{2}}+1\right| .
$$

What follows is:

$$
\left|2 * 2^{-s}-1\right|=\left|-2 * 2^{-\delta 2}+1\right|
$$

Then:

(A) $2 * 2^{-s}-1=-2 * 2^{-\delta 2}+1$ or

(B) $2 * 2^{-s}-1=2 * 2^{-\delta_{2}}-1$.

From A, we get:

$$
\begin{aligned}
& 2^{1-s}-1=-2^{1-\delta_{2}}+1, \\
\therefore \quad & 2^{1-\delta 2}=-2^{1-s}+2, \\
\therefore \quad & 2^{\delta_{2}}=\frac{2}{2-2^{1-s}}, \\
\therefore & 2^{\delta_{2}}=\frac{1}{1-2^{-s}} .
\end{aligned}
$$

A then leads to our $s$ being replaced with $\log _{2}\left(\frac{1}{1-2^{-s}}\right)$ and B leads to our replacement being $s$. 
Because both differences should be non-negative (we are always doing the limiting line of the extension minus the limiting line of convexity), only $\mathrm{A}$ is a valid result.

It is then the case that $\delta_{2}=\log _{2}\left(\frac{1}{1-2^{-s}}\right)$.

With this, our function is smooth of class $C^{\infty}$ ([5], p. 511).

Once more, to calculate our perimeters, we have to use the formula we had for the negative case and the new formula, which will be a result of our calculations.

Assume that $A=1$.

$$
\begin{aligned}
f\left(\lambda_{s c n}\right)= & (1-\lambda)^{\log _{2}\left(\frac{1}{1-2^{-s}}\right)}-\lambda^{\log _{2}\left(\frac{1}{1-2^{-s}}\right)} \\
f^{\prime}\left(\lambda_{s c n}\right)= & \log _{2}\left(\frac{1}{1-2^{-s}}\right)(1-\lambda)^{\log _{2}\left(\frac{1}{1-2^{-s}}\right)-1} \\
& -\log _{2}\left(\frac{1}{1-2^{-s}}\right) \lambda^{\log _{2}\left(\frac{1}{1-2^{-s}}\right)-1}, \\
\left(f^{\prime}\left(\lambda_{s c n}\right)\right)^{2}= & \left(\log _{2}\left(\frac{1}{1-2^{-s}}\right)(1-\lambda)^{\log _{2}\left(\frac{1}{1-2^{-s}}\right)-1}\right. \\
& \left.-\log _{2}\left(\frac{1}{1-2^{-s}}\right) \lambda^{\log _{2}\left(\frac{1}{1-2^{-s}}\right)-1}\right)^{2}, \\
p \equiv & \int_{0}^{1}\left(1+\left(\log _{2}\left(\frac{1}{1-2^{-s}}\right)^{\left.\log _{2}(1-\lambda)^{\frac{1}{1-2^{-s}}}\right)-1}\right.\right. \\
& \left.\left.-\log _{2}\left(\frac{1}{1-2^{-s}}\right) \lambda^{\log _{2}\left(\frac{1}{1-2^{-s}}\right)-1}\right)^{2}\right)^{\frac{1}{2}} d \lambda .
\end{aligned}
$$

Let's call this new set of functions New Negative.

Our new tables ${ }^{2}$, for the cases in which our functions are $f(x)=1$ and $f(x)=-1$, just to exemplify, are:

\footnotetext{
2 The first value for Arc Length in the table has been attained through simple substitution in the formula. The second and the third values have been attained by means of the formula for circumference length (average between vertical and horizontal values).
} 
New Positive System

$$
f\left(\lambda_{s c p}\right)=(1-\lambda)^{\log _{2}\left(\frac{1}{1-2^{-\frac{1}{s}}}\right)} A+\lambda^{\log _{2}\left(\frac{1}{1-2^{-\frac{1}{s}}}\right)} A
$$

for when $|f|=f$ and

$$
f\left(\lambda_{s c n}\right)=-(1-\lambda)^{\frac{1}{s}} A-\lambda^{\frac{1}{s}} A
$$

for when $|f|=-f$.

Table 1: $\mathrm{f}(\mathrm{x})=1$

\begin{tabular}{cc}
\hline \hline$s$ & Arc Length \\
\hline 1 & 1 \\
0.5 & 1.57 \\
0.25 & 2.16 \\
\hline \hline
\end{tabular}

New Negative System

$$
f\left(\lambda_{\text {scp }}\right)=(1-\lambda)^{s} A+\lambda^{s} A
$$

for when $|f|=f$ and

$$
f\left(\lambda_{s c n}\right)=-(1-\lambda)^{\log _{2}\left(\frac{1}{1-2^{-s}}\right)} A-\lambda^{\log _{2}\left(\frac{1}{1-2^{-s}}\right)} A
$$

for when $|f|=-f$.

Table 2: $\mathrm{f}(\mathrm{x})=-1$

\begin{tabular}{cc}
\hline \hline$s$ & Arc Length \\
\hline 1 & 1 \\
0.5 & 1.43 \\
0.25 & 1.85 \\
\hline \hline
\end{tabular}




\section{Interval of Interest}

For the $s$ in $S$-convexity to make sense, we should probably stick to the New Negative System. In this case, no better exponent should be found.

We then have $1 / s$ providing us with a nicer shape, and perhaps making us have less work with the formula by the time of applying it, and the $\delta_{2}$ we have found here, which gives us the so expected equal distance for both pieces of the definition of the phenomenon (non-negative and negative).

If we stick to what we had before, we are farther from what we expect in Mathematics, so that we here suggest that we now use the New Negative System as a definition for $S$-convexity.

\section{Conclusion}

We have refined the definition of the phenomenon $S$-convexity once more.

This time, it is about the distance between the limiting line of the extension of convexity and the limiting line of convexity being the same in both pieces of our definition (the non-negative functions form one piece and the negative functions form another).

We have decided to deal with the phenomenon $S$-convexity as if it were an exclusively extensional concept for issues that have to do with practicality and accuracy (we now forbid $s$ to assume the value 1 in our definition).

We have extended the domain of the $S$-convex functions to $\Re$ because the definition should only impose limitations to the image of the functions, not to the domain.

We have decided to swap the coefficients in our definition because $\lambda=0$ should bring $f(x)$ to life, not $f(x+\delta)$.

We have added the interval of definition of $s$ to our geometric definition to make it be independent from the analytical definition.

Based on our new findings and decisions, we produce a new update in our definition for the phenomenon $S$-convexity, update that we present below.

\section{Analytical Definition}

Definition 9. A function $f: X \rightarrow \Re$, where $|f(x)|=f(x)$, is told to belong to $K_{s}^{2}$ if the inequality

$$
f((1-\lambda) x+\lambda(x+\delta)) \leq(1-\lambda)^{s} f(x)+\lambda^{s} f(x+\delta)
$$

holds $\forall \lambda / \lambda \in[0,1] ; \forall x / x \in X ; s=s_{2} / 0<s_{2}<1 ; X / X \subseteq \Re \wedge X=[a, b]$; $\forall \delta / 0<\delta \leq(b-x)$. 
Definition 10. A function $f: X \rightarrow \Re$, where $|f(x)|=-f(x)$, is told to belong to $K_{s}^{2}$ if the inequality

$$
f((1-\lambda) x+\lambda(x+\delta)) \leq(1-\lambda)^{\log _{2}\left(\frac{1}{1-2^{-s}}\right)} f(x)+\lambda^{\log _{2}\left(\frac{1}{1-2^{-s}}\right)} f(x+\delta)
$$

holds $\forall \lambda / \lambda \in[0,1] ; \forall x / x \in X ; s=s_{2} / 0<s_{2}<1 ; X / X \subseteq \Re \wedge X=[a, b]$; $\forall \delta / 0<\delta \leq(b-x)$.

Remark 2. If the inequalities are obeyed in the reverse situation by $f$, then $f$ is said to be $s_{2}$-concave.

\section{Geometric Definition}

Definition 11. A real function $f: X \rightarrow Y$, for which $|f(x)|=f(x)$, is called $S$-convex ${ }^{3}$ if and only if, for all choices $\left(x_{1} ; y_{1}\right)$ and $\left(x_{2} ; y_{2}\right)$, where $\left\{x_{1}, x_{2}\right\} \subset X,\left\{y_{1}, y_{2}\right\} \subset Y, Y=I m_{f}, x_{1} \neq x_{2}$, and $y_{1} \neq y_{2}$, it happens that the line drawn between $\left(x_{1} ; y_{1}\right)$ and $\left(x_{2} ; y_{2}\right)$ by means of the expression $(1-\lambda)^{s} y_{1}+\lambda^{s} y_{2}$, where $\lambda \in[0,1]$, does not contain any point with height, measured against the vertical Cartesian axis, that is inferior to the height of its horizontal equivalent in the curve representing the ordered pairs of $f$ in the interval considered for the line in terms of distance from the origin of the Cartesian axis.

Definition 12. A real function $f: X \rightarrow Y$, for which $|f(x)|=-f(x)$, is called $S$-convex ${ }^{4}$ if and only if, for all choices $\left(x_{1} ; y_{1}\right)$ and $\left(x_{2} ; y_{2}\right)$, where $\left\{x_{1}, x_{2}\right\} \subset X,\left\{y_{1}, y_{2}\right\} \subset Y, Y=I m_{f}, x_{1} \neq x_{2}$, and $y_{1} \neq y_{2}$, it happens that the line drawn between $\left(x_{1} ; y_{1}\right)$ and $\left(x_{2} ; y_{2}\right)$ by means of the expression $(1-\lambda)^{\log _{2}\left(\frac{1}{1-2^{-s}}\right)} y_{1}+\lambda^{\log _{2}\left(\frac{1}{1-2^{-s}}\right)} y_{2}$, where $\lambda \in[0,1]$, does not contain any point with height, measured against the vertical Cartesian axis, that is inferior to the height of its horizontal equivalent in the curve representing the ordered pairs of $f$ in the interval considered for the line in terms of distance from the origin of the Cartesian axis.

Remark 3. If all the points defining the function are located above the limiting line instead, then $f$ is called $S$-concave.

\footnotetext{
${ }^{3} \mathrm{~S}$ must be replaced, as needed, with a fixed constant located between 0 and 1 but different from 0 and 1. For instance, if the chosen constant is 0.5 , then the function will be 0.5 -convex or $\frac{1}{2}$-convex and $\mathrm{S}$ will be 0.5 in the expression that defines the limiting line.

${ }^{4} \mathrm{~S}$ must be replaced, as needed, with a fixed constant located between 0 and 1 but different from 0 and 1. For instance, if the chosen constant is 0.5 , then the function will be 0.5 -convex or $\frac{1}{2}$-convex and $\mathrm{S}$ will be 0.5 in the expression that defines the limiting line.
} 


\section{References}

[1] M.R. Pinheiro, First note on the definition of $S_{2}$-convexity, Advances in Pure Mathematics, 1 (2011), 1-2, doi: 10.4236/apm.2011.11001.

[2] M.R. Pinheiro, Minima domain intervals and the S-convexity, as well as the convexity, phenomenon, Advances in Pure Mathematics, 2, No. 6 (2012), 457-458, doi: 10.4236/apm.2012.26069.

[3] M.R. Pinheiro, First note on the shape of S-convexity, International Journal of Pure and Applied Mathematics, 90, No. 1 (2014), 101-107, doi: 10.12732/ijpam.v90i1.12.

[4] M.R. Pinheiro, Second note on the shape of S-convexity, International Journal of Pure and Applied Mathematics, 92, No. 2 (2014), 297-303, doi: 10.12732/ijpam.v92i2.11.

[5] E.V. Shikin, Handbook and Atlas of Curves, CRC Press (1995), ISBN: 9780849389634 . 
